\title{
Capacitación en diagnóstico e intervención psicopedagógica de la dislalia funcional
}

\author{
Training in diagnosis and psychopedagogical intervention of functional \\ dyslalia
}

\author{
Melvy Roxana Mamani LLanque \\ melvymllanque@gmail.com
}

Universidad Mayor Real y Pontificia de San Francisco Xavier de Chuquisaca, Sucre Bolivia

\section{RESUMEN}

La dislalia es una alteración del lenguaje oral que consiste en una inadecuada pronunciación de las palabras, los problemas de dislalia son comunes en los niños menores de siete años, estas dificultades afectan la capacidad de expresión verbal y por ende la comunicación. En el Jardín Infantil Acción Social "A" se diagnosticó que los niños muestran dificultades en la pronunciación de palabras y las maestras que se desempeñan en este centro educativo no poseen las competencias suficientes para la atención de la dislalia. En consecuencia esta investigación se propuso diseñar un programa de capacitación en diagnóstico e intervención de la dislalia funcional que le permitan desarrollar procesos de estimulación del lenguaje en su componente fonológico de sus estudiantes. La muestra de tipo intencional quedó conformada por 32 niños y 6 maestras. Se elaboró un diagnóstico de las competencias que poseían las docentes con respecto al diagnóstico e intervención de la dislalia, así como también de las dificultades específicas de pronunciación de cada niño. Con base a los resultados se diseñó el programa de capacitación para resolver las necesidades encontradas. El programa fue evaluado por el Método Delphi el cual obtuvo como resultado del criterio de los expertos como "MUY ADECUADO" y por lo tanto se recomienda su aplicación.

Palabras clave: Dislalia; capacitación docente; educación inicial; programa de capacitación, método Delphi

\section{ABSTRACT}

The dyslalia is an alteration of the oral language that consists of an inadequate pronunciation of the words, the problems of dyslalia are common in the children under seven years, these difficulties affect the capacity of verbal expression and therefore the communication. In the Kindergarten Social Action "A" it was diagnosed that children show difficulties in the pronunciation of words and the teachers who work in this educational center do not have sufficient skills for the attention of dyslalia. Consequently, this research set out to design a training program in diagnosis and intervention of functional dyslalia that allow it to develop language stimulation processes in its phonological component of its students. The intentional sample was made up of 32 children and 6 teachers. A diagnosis was made of the competencies that teachers possessed regarding the diagnosis and intervention of dyslalia, as well as the specific pronunciation difficulties of each child. Based on the results, the training program was designed to meet the needs found. The program was evaluated by the Delphi Method which obtained as a result of the experts' criteria as "VERY SUITABLE" and therefore its application is recommended.

Key words: Dyslalia; Teacher training; Initial Education; Training program; Delphi Method 
INTRODUCCIÓN

El término dislalia etimológicamente, significa hablar mal o hablar con dificultad. La dislalia es la alteración del lenguaje oral, que consiste en una mala pronunciación o articulación de las palabras, como consecuencia de una dificultad para pronunciar uno o varios fonemas. Es ocasionada por alteraciones funcionales de los órganos periféricos del habla: lengua, labios, paladar, mejillas y dientes; sin que haya lesiones físicas o malformaciones. (Peña Casanova, 2005) Puede afectar a cualquier consonante o vocal y son muy frecuentes en la infancia, sobre todo en los primeros años escolares con una adecuada intervención la mayoría de ellas desaparecen. Pero si no se atiende adecuadamente el lenguaje del niño puede hacerse ininteligible, por las desfiguraciones verbales que emplea continuamente (Pascual, 2004).

La mayoría de los defectos dislálicos que presentan los niños no son debido a causas anatómicas, ni las lesiones nerviosas; más bien son un problema de educación (Lima, 2004). En algunos casos el niño dislálico tiene conciencia de su inadecuada articulación e intenta corregirse imitando la articulación correcta del adulto, pero sus órganos no responden y no encuentra el movimiento correcto que debe realizar para pronunciar un sonido correctamente (Alonzo, 2010).

Los problemas de dislalia son muy comunes en los niños menores de siete años, estos afectan la capacidad de expresión verbal y por ende la comunicación. Esta es la edad que los niños acuden al jardín de infantes para iniciar su educación y socialización en el ambiente escolar. Se puede afirmar a que la dislalia dificulta la comunicación del niño, afectando a otros ámbitos de su desarrollo como la seguridad, confianza, autoestima, aprendizaje, desarrollo de la personalidad, adaptación social y rendimiento escolar en general. (Castejón y Navas, 2011). Esta es la razón por la cual, desde el nivel inicial la manifestación de este tipo de dificultades requiere de la atención y el ajuste de la acción psicopedagógica.

A pesar de que la dislalia es muy común en la educación inicial es poco lo que se hace para diagnosticar y tratar este problema; esto trae como consecuencia que los niños demoren más tiempo en superar sus errores en la pronunciación de fonemas, incluso llegando a la educación primaria con estas dificultades que pudieron ser atendidas y resueltas en el nivel inicial.

Pascual (2004) recomienda que al presentarse un caso de dislalia es indispensable movilizar las competencias de un equipo interdisciplinario capaz de establecer dentro de las mejores condiciones, un diagnóstico confiable y que asegure una atención precoz y oportuna.

Sin embargo, ante la ausencia de este equipo interdisciplinario en las instituciones educativas, los maestros son los que están llamados para asumir este compromiso, para lo cual deben tener las competencias en el diagnóstico, así como también en estrategias para intervenir y en casos muy severos sugerir a los padres para acudir a un especialista. De esta manera se pueden atender los casos de dislalia que se presenten en sus cursos. Una atención temprana y adecuada le permitirá a los estudiantes una mejor comunicación y un adecuado desarrollo psicosocial. (Jiménez y Obispo, 2006).

En el Jardín Infantil Acción Social "A" (JIASA) se observaron algunos casos de problemas de pronunciación y las maestras no tienen información de cómo atender esta situación. Por lo expuesto anteriormente, se plantea la propuesta de un programa de capacitación para las maestras, en diagnóstico e intervención psicopedagógica de la dislalia funcional. El desarrollo de este programa 
beneficiaría al personal docente ya que le suministra las herramientas específicas para generar cambios en la metodología y herramientas de estimulación para el desarrollo del lenguaje oral de los niños. Este programa también tendrá un impacto positivo en la calidad del servicio educativo que el Jardín Infantil Acción Social "A" brinda a la ciudadanía; siendo los principales beneficiarios todos los niños que asisten a esta unidad educativa pero principalmente los niños que presentan algún problema de pronunciación. Otro beneficio es el obtenido por los padres de familia que contarán con acompañamiento de mejor calidad por parte de la maestra, recibiendo recomendaciones acerca de actividades que podrían desarrollar en casa para apoyar el desarrollo de la pronunciación en sus hijos.

Este trabajo tiene el respaldo legal de la Ley de Educación vigente en Bolivia la cual estipula que la educación inicial en familia comunitaria, escolarizada debe estimular el desarrollo de capacidades y habilidades lingüísticas. De la misma forma las maestras del Jardín Infantil Acción Social están motivadas para desarrollar el programa de capacitación en Dislalia funcional.

Por las consideraciones señalada este estudio tuvo como propósito diseñar un programa de capacitación en diagnóstico e intervención de la dislalia funcional dirigido a maestras del JIASA para desarrollar procesos de estimulación del lenguaje en su componente fonológico en niños con dislalia. El cual se compartirá en este artículo a fin de contribuir con la divulgación del estudio.

\section{MÉTODO}

El estudio se llevó a cabo bajo una investigación proyectiva, ya que su objetivo fue diseñar un programa como solución a un problema a partir de un diagnóstico de necesidades. (Hurtado, 2012). Para lo cual se identifican las necesidades y se definen las variables, se comprenden las causas $\mathrm{y}$ consecuencias, se identifican posibles soluciones y se elabora una propuesta capaz de producir los cambios.

Se aplicó el método Delphi o consenso de panel el cual consiste en una consulta a expertos que permite la validación teórica del modelo o propuesta de investigación (Almenara y Moro, 2014). Este método se utilizó al final de la investigación para demostrar la validez de la propuesta que fue diseñada como respuesta al problema.

El diseño es de campo ya que se toman los datos directamente de los sujetos investigados en su ambiente natural donde ocurren los hechos, sin manipulación de variables. (Hernández, Fernández y Baptista, 2010).

La población estuvo conformada por 99 niños (as) de los tres paralelos "A", "B" y "C" de segunda sección de Educación Inicial en Familia Comunitaria, Escolarizada del JIASA de la ciudad de Sucre y las seis maestras que atienden a estos grupos de estudiantes.

La técnica de muestreo que se utilizó en esta investigación es no probabilística llamada también intencionada o dirigida, en las muestras de este tipo la elección de los sujetos depende de la decisión que toma el investigador en función del tiempo, los recursos y las posibilidades de acceso que tiene a la población. (Hurtado de Barrera, 2012).

Se tomó como muestra a los 32 niños (as) del paralelo " $A$ " de segunda sección de Educación Inicial en Familia Comunitaria, Escolarizada del JIASA de la ciudad de Sucre y también se tomó en cuenta a las seis maestras que se desempeñan en este jardín de niños. La edad de los niños estuvo comprendida entre 5 años y 5 años y 11 meses.

El instrumento que se aplicó para la recogida de información fue el cuestionario 
constituido por un listado de preguntas escritas y previamente analizadas, este instrumento fue aplicado a las maestras del nivel inicial con el fin de recabar información sobre los conocimientos que las maestras poseen sobre cómo diagnosticar, atender y prevenir la dislalia funcional. Está constituido por 11 ítems que corresponden a las dimensiones diagnóstico e intervención.

Otro instrumento fue el Test para evaluar Procesos de Simplificación Fonológica (TEPROSIF-R) desarrollado por especialistas lingüistas y fonoaudiólogos. (Pavez, Magiolo y Colomba, 2009). Este test permite identificar los niños que tienen problemas de pronunciación, se ha utilizado ampliamente en Chile y algunas experiencias en Argentina.

El test establece tres niveles de desempeño fonológico que son: Desempeño normal, de riesgo y deficiente; los parámetros del número de errores que se considera como normal, riesgo o deficiente varia ya que toma en cuenta la edad del niño al que es aplicado el test. La presente investigación utilizó los parámetros que están establecidos para niños de 5 años a 5 años y 11 meses ya que todos los niños a los que se aplicó el test se encontraban dentro de este rango de edad.

Este test ha sido sometido a estudios rigoroso de validez y confiabilidad a través de la aplicación del instrumento a una muestra amplia de niños de 3-6 años con diferentes, niveles socioeconómicos, características y en diferentes regiones, lo que permitió realizar estudios estadísticos que comprueban la validez y confiabilidad del instrumento.

Se aplicó el instrumento TEPROSIF-R a los estudiantes de la segunda sección de Educación inicial en Familia Comunitaria, Escolarizada del Jardín de Infancia Acción Social "A" para evaluar el desarrollo fonológico de los mismos. Esta evaluación permitió detectar los tipos y magnitud de problemas de pronunciación en el nivel inicial.
El test consta de 37 ítems que son palabras que tienen diferente complejidad silábica. Así se incluyen palabras monosílabas, bisílabas, trisílabas y polisílabas con acentuación y estructuras silábicas diferentes.

El test se aplica con ayuda de 39 láminas de la cuales las dos primeras son ejemplos para familiarizar al niño con la actividad, cada lamina contiene dos dibujos en la parte superior aparece un dibujo para presentar la palabra estímulo y en la parte inferior hay otro dibujo que se usa para que el niño emita la palabra deseada completando con ella una oración. Es importante registrar la producción del niño tal como lo pronunció ya que esto facilitará el análisis e interpretación del desempeño del niño. (Moreno y Ramírez, 2012).

El test mide tres dimensiones: Estructura, Asimilación y Sustitución según (Pavez, Magiolo y Colomba, 2009). Procesos relacionados con la Estructura (E) de la palabra es cuando el niño simplifica las sílabas transformándolas en estructuras del tipo consonante + vocal (CV) para facilitar su emisión.

Procesos de la Asimilación (A) son procedimientos mediante los cuales se cambia un fonema para hacerlo igual o semejante a otro presente en la palabra modelo o en la emitida por el niño.

Procesos de sustitución (S) son procedimientos para simplificar la palabra donde se sustituyen fonemas pertenecientes a una clase por miembros de otra clase; o fonemas de una misma clase entre sí.

RESULTADOS

En primer lugar se presentan los resultados de la aplicación del Test para evaluar Procesos de Simplificación Fonológica aplicado a niños del Jardín de Infancia Acción Social "A". 


\section{Dificultades de pronunciación}

De los 32 niños (as) a los que se les aplicó el test solo uno, que representa el 3\%, no presenta dificultades de pronunciación y el 97\% restante comete algún tipo de error en la pronunciación. Este resultado coincide con lo planteado por Aguilera (2004), quien señala que no se pueden catalogar dificultades del aprendizaje aquellas características que forman parte del desarrollo evolutivo o biofisiológico.

La mayoría de los niños comete algún tipo de error en la pronunciación pero eso no significa que todos estos niños presentan un problema serio, sino que dentro de este número de niños están niños con un desarrollo fonológico normal, niños que presentan riesgo y también se encuentra los que presentan serios problemas de pronunciación.

Tabla 1. Frecuencia de las dificultades de pronunciación

\begin{tabular}{ccc}
\hline \multirow{2}{*}{ Indicadores } & \multicolumn{2}{c}{ Frecuencia } \\
& Absoluta No & Relativa \% \\
\hline Sin dificultades & 1 & $3 \%$ \\
Con dificultades & 31 & $97 \%$ \\
\hline Total & $\mathbf{3 2}$ & $\mathbf{1 0 0 \%}$ \\
\hline
\end{tabular}

\section{Desempeño fonológico general}

El 81\% de la muestra en estudio presenta nivel normal de desempeño fonológico, el $13 \%$ se ubica en el nivel de riesgo y el $6 \%$ presenta un nivel deficiente.

La mayoría de los niños comete una cantidad de errores que es considerada normal de acuerdo a lo esperado para su edad y desarrollo biológico. El 13\% de los niños muestran retraso en el desarrollo fonológico que en ocasiones puede pasar desapercibido y un porcentaje menor el $6 \%$ presenta un marcado retraso fonológico.

Tabla 2. Desempeño fonológico general

\begin{tabular}{lcc}
\hline \multicolumn{1}{c}{ Indicadores } & \multicolumn{2}{c}{ Frecuencia } \\
& Absoluta No & Relativa \% \\
\hline Normal de 0 a 14 errores & 26 & $81 \%$ \\
Riesgo de 15 a 21 errores & 4 & $13 \%$ \\
Deficiente de 22 a más errores & 2 & $6 \%$ \\
\hline \multicolumn{1}{c}{ Total } & $\mathbf{3 2}$ & $\mathbf{1 0 0 \%}$ \\
\hline
\end{tabular}




\section{Procesos relacionados con la Estructura silábica y la palabra}

De los 32 niños a los que se les aplicó el test, solo uno, que representa el $3 \%$ de la muestra, supera completamente las dificultades de pronunciación relacionadas con la estructura silábica y de la palabra y el resto que representa el $97 \%$ de los niños comete alguno o varios errores de este tipo.

Tabla 3. Dificultades de pronunciación relacionados con la estructura silábica y de la palabra

\begin{tabular}{ccc}
\hline Indicadores & \multicolumn{2}{c}{ Frecuencia } \\
& Absoluta No & Relativa \% \\
\hline Si supera & 1 & $3 \%$ \\
No supera & 31 & $97 \%$ \\
\hline Total & $\mathbf{3 2}$ & $\mathbf{1 0 0 \%}$ \\
\hline
\end{tabular}

Desempeño fonológico relacionado con la estructura silábica y de la palabra

El $88 \%$ de la muestra tiene un desempeño fonológico normal según el total de procesos relacionados con la estructura silábica y la palabra, un $9 \%$ se ubica en el nivel de riesgo y un $3 \%$ presenta un desempeño deficiente.
La mayoría de los niños comete de 0 a 8 errores que afectan la metria de la palabra y silaba esta cantidad es considerada normal, sin embargo también hay niños en riesgo y un porcentaje mínimo presenta retraso fonológico con relación a la estructura fonológica.

Tabla 4. Nivel de desempeño fonológico relacionado con la estructura silábica y de la palabra

\begin{tabular}{ccc}
\hline Indicadores & \multicolumn{2}{c}{ Frecuencia } \\
& Absoluta No & Relativa \% \\
\hline Normal de 0 a 8 errores & 28 & $88 \%$ \\
Riesgo de 9 a 12 error & 3 & $9 \%$ \\
Deficiente de 13 a más errores & 1 & $3 \%$ \\
\hline Total & $\mathbf{3 2}$ & $\mathbf{1 0 0 \%}$ \\
\hline
\end{tabular}

Errores de pronunciación relacionados con la estructura silábica y de la palabra

De los 32 niños (as) a los que se les aplico de test, dan como resultado que el $47 \%$ comete errores en la pronunciación de grupos consonánticos y un $53 \%$ no comete; $25 \%$ reduce los diptongos y $75 \%$ los pronuncia correctamente; $66 \%$ omite consonantes trabantes y $34 \%$ no comete este tipo error; $88 \%$ Omite elementos átonos y $13 \%$ no las omite; $9 \%$ omite elementos tónicos y 91\% no omite este tipo de elementos; y por ultimo un $28 \%$ añade algún fonema y un $72 \%$ no comete este error.

Estos resultados muestran que Los niños cometen errores que afectan directamente la estructura de la palabra y silaba en su mayoría reduciendo las metria del mismo al omitir elementos átonos y tónicos, al reducir diptongos, grupos consonánticos y trabantes y es un porcentaje mínimo aumenta la metria de la palabra. 
Tabla 5. Errores relacionados con la estructura silábica y la palabra

\begin{tabular}{lccccc}
\hline \multicolumn{1}{c}{ Indicadores } & & \multicolumn{2}{c}{ Frecuencia } & \multicolumn{2}{c}{ TOTAL } \\
& & Absoluta No & Relativa \% & \multicolumn{2}{c}{ TOT } \\
\hline E.1 Reducción de grupos & Si supera & 15 & $47 \%$ & \multirow{2}{*}{32} & \multirow{2}{*}{$100 \%$} \\
consonánticos & No supera & 17 & $53 \%$ & & \\
E.2 Reducción de diptongos & Si supera & 8 & $25 \%$ & 32 & $100 \%$ \\
& No supera & 24 & $75 \%$ & & \\
E.3 Omisión de consonantes & Si supera & 21 & $66 \%$ & 32 & $100 \%$ \\
trabantes & No supera & 11 & $34 \%$ & & \\
E.4 Omisión de elementos & Si supera & 28 & $88 \%$ & 32 & $100 \%$ \\
átonos & No supera & 4 & $13 \%$ & & \\
E.5 Omisión de elementos & Si supera & 3 & $9 \%$ & 32 & $100 \%$ \\
tónica & No supera & 29 & $91 \%$ & & \\
E.6Adición de fonemas o & Si supera & 9 & $28 \%$ & 32 & $100 \%$ \\
silabas & No supera & 23 & $72 \%$ & &
\end{tabular}

\section{Desempeño fonológico relacionado con los procesos de asimilación}

Según errores cometidos con relación a procesos de asimilación el 85\% tienen un desempeño fonológico normal, mientras que un $9 \%$ presenta riesgo y un $6 \%$ presenta un desempeño deficiente.
En su mayoría los niños cometen errores al remplazar un fonema por otro que está presente en la palabra en una cantidad que es considerada normal, sin embargo también hay niños en riesgo y un porcentaje menor presenta retraso fonológico cometiendo más errores de este tipo.

Tabla 6. Nivel de desempeño fonológico y los procesos de asimilación

\begin{tabular}{lcc}
\hline \multicolumn{1}{c}{ Indicadores } & \multicolumn{2}{c}{ Frecuencia } \\
& Absoluta & Relativa \\
& $\mathbf{N o}$ & $85 \%$ \\
\hline Normal de 0 a 4 errores & 27 & $9 \%$ \\
Riesgo de 5 a 6 errores & 3 & $6 \%$ \\
Deficiente de 7 a más errores & 2 & $\mathbf{1 0 0 \%}$ \\
\hline Total & $\mathbf{3 2}$ & \\
\hline
\end{tabular}

\section{Desempeño fonológico relacionado con los procesos de sustitución}

Según los errores cometidos por procesos de sustitución el $81 \%$ tienen un desempeño fonológico normal, mientras que un $13 \%$ presenta riesgo y un $6 \%$ presenta un desempeño deficiente.

En su mayoría los niños comete errores de remplazar unos fonemas por otro que no se parecen a ninguno de los fonemas presentes en la palabra en una cantidad que es considerada normal, sin embargo también hay niños en riesgo y un porcentaje menor presenta retraso fonológico según este tipo de error.

A continuación se presentan los resultados del cuestionario aplicado a las maestras del jardín de Infancia Acción Social "A". 
Tabla 7. Nivel de desempeño fonológico y los procesos de sustitución

\begin{tabular}{lcc}
\hline \multicolumn{1}{c}{ Indicadores } & \multicolumn{2}{c}{ Frecuencia } \\
& Absoluta № & Relativa \% \\
\hline Normal de 0 a 4 errores & 26 & $81 \%$ \\
Riesgo de 5 a 6 errores & 4 & $13 \%$ \\
Deficiente de 7 a más errores & 2 & $6 \%$ \\
\hline \multicolumn{1}{c}{ Total } & $\mathbf{3 2}$ & $\mathbf{1 0 0 \%}$ \\
\hline
\end{tabular}

\section{Dimensión conocimiento}

El 83\% de la muestra de maestras declara que su conocimiento es insuficiente en cuanto al tratamiento y prevención de dislalias funcionales, un $17 \%$ afirma que su conocimiento al respecto es nulo. Estos resultados evidencian la necesidad de generar un programa para la capacitación de las maestras en referencia a la dislalia.

Tabla 8. Conocimiento sobre dislalia

\begin{tabular}{lcc}
\hline Indicadores & \multicolumn{2}{c}{ Frecuencia } \\
& Absoluta No & Relativa \% \\
\hline Suficiente & $\mathbf{0}$ & $\mathbf{0 \%}$ \\
Insuficiente & $\mathbf{5}$ & $\mathbf{8 3 \%}$ \\
Nulo & $\mathbf{1}$ & $\mathbf{1 7 \%}$ \\
Total & 6 & $\mathbf{1 0 0 \%}$ \\
\hline
\end{tabular}

Dimensión Intervención aplicación de técnicas para el mejoramiento de la pronunciación

De las seis maestras de la muestra, cinco declaran que no realizan actividades específicas para mejorar el nivel fonológico de los niños. Tienen la necesidad de recibir capacitación en actividades específicas para la atención de las diferentes dificultades como: la discriminación auditiva, movilidad de labios y lengua, control de la respiración, pronunciación de fonemas. Estos resultados sustentan la necesidad de diseñar un programa que permita capacitar a las maestras en diagnóstico e intervención de la dislalia para promover los cambios en los procesos de estimulación del lenguaje en su componente fonológico en los niños del Jardín de Infantes. 
Tabla 9. Ejecución de actividades para el mejoramiento de la pronunciación

\begin{tabular}{lcc}
\hline Indicadores & \multicolumn{2}{c}{ Frecuencia } \\
& Absoluta No & Relativa \% \\
\hline Suficiente & 1 & $17 \%$ \\
Insuficiente & 2 & $33 \%$ \\
Nulo & 3 & $50 \%$ \\
Total & $\mathbf{6}$ & $\mathbf{1 0 0 \%}$ \\
\hline
\end{tabular}

\section{Programa de capacitación en diagnóstico} e intervención psicopedagógica de la dislalia

\section{Fundamentación pedagógica}

El programa de capacitación se fundamenta en el constructivismo que es una corriente pedagógica basada en la teoría del conocimiento constructivista, que postula que el conocimiento es una construcción del ser humano, centrado en el estudiante y sus experiencias previas y postula la necesidad de entregar al estudiante herramientas o andamiajes que le permitan construir su propio procedimiento para resolver una situación problemática, lo que implica que sus ideas se modifiquen y siga aprendiendo (Ausubel, Novak y Hanesian, 1997).

El constructivismo educativo propone un paradigma donde el proceso de enseñanza se percibe y se lleva a cabo como un proceso dinámico, participativo e interactivo del sujeto, de modo que el conocimiento sea una auténtica construcción operada por la persona que aprende.

\section{Fundamentación Legal}

Las bases legales de la propuesta son los Artículos 7, 59 y 80 de la Constitución Política de Bolivia por el Estado Plurinacional de Bolivia en el 2009 y los Artículos 21 y 22 de Ley de la educación "Avelino Siñani Elizardo Pérez"

\section{Perfil del Usuario}

Este programa de capacitación está dirigido al personal docente de Educación Inicial

\section{Perfil del Facilitador}

El facilitador debe ser un especialista en psicopedagogía o lingüista fonoaudiólogo.

\section{Organización}

El programa está constituido por las siguientes partes: introducción, justificación, características, perfil del usuario, objetivos, estructura, metodología, recursos y medios didácticos, procesos de evaluación. 
Tabla 10. Estructura

\begin{tabular}{|c|c|c|c|}
\hline Unidad temática & Temas & Tiempo & Total \\
\hline Unidad temática № 1 & Tema № 1 & & \multirow{3}{*}{6 horas } \\
\hline El lenguaje oral & El lenguaje oral infantil & 3 horas & \\
\hline $\begin{array}{l}\text { infantil y los } \\
\text { trastornos habla. }\end{array}$ & Tema № 2 trastornos del habla. & 3 horas & \\
\hline & № 3 & \multirow{2}{*}{3 horas } & \multirow{4}{*}{5 horas } \\
\hline № 2 La dislalia & La dislalia funcional & & \\
\hline funcional y su & № 4 & & \\
\hline $\begin{array}{l}\text { influencia en el } \\
\text { desarrollo del niño }\end{array}$ & $\begin{array}{l}\text { Influencia de la dislalia } \\
\text { funcional en el desarrollo del } \\
\text { niño }\end{array}$ & 2 horas & \\
\hline $\begin{array}{l}\text { № } 3 \text { Diagnostico de la } \\
\text { dislalia funcional }\end{array}$ & $\begin{array}{l}\text { № } 5 \\
\text { Diagnóstico de la dislalia } \\
\text { funcional } \\
\text { № } 6\end{array}$ & 4 horas & 2 horas \\
\hline \multirow{3}{*}{$\begin{array}{l}\text { № } 4 \text { Intervención } \\
\text { indirecta para el } \\
\text { desarrollo del } \\
\text { lenguaje oral. }\end{array}$} & $\begin{array}{l}\text { Estimulación de las bases } \\
\text { bucofonatorias }\end{array}$ & 4 horas & \multirow{3}{*}{16 horas } \\
\hline & $\begin{array}{l}\text { № } 7 \\
\text { respiración y soplo. } \\
\text { № } 8\end{array}$ & 4 horas & \\
\hline & $\begin{array}{l}\text { Estimulación de la percepción y } \\
\text { discriminación auditiva } \\
\text { № } 9\end{array}$ & 4 horas & \\
\hline $\begin{array}{l}\text { № } 5 \text { Intervención } \\
\text { directa para el }\end{array}$ & $\begin{array}{l}\text { Enseñanza y adquisición de la } \\
\text { articulación correcta de los } \\
\text { fonemas }\end{array}$ & 4 horas & \multirow{3}{*}{8 horas } \\
\hline desarrollo del & № 10 & & \\
\hline lenguaje oral & $\begin{array}{l}\text { Automatización y } \\
\text { generalización de la } \\
\text { articulación correcta }\end{array}$ & 4 horas & \\
\hline
\end{tabular}

Cada tema está planificado con los objetivos de aprendizaje, el contenido discriminado en tres sistemas: de conocimiento, habilidades y valores. Describe las orientaciones metodológicas para el facilitador y el participante; los medios y recursos didácticos adecuados, contempla las características de las técnicas que permiten evaluar el logro alcanzado al desarrollar cada tema.

El programa de capacitación en diagnostico e intervención de dislalias funcionales fue diseñado tomando en cuenta las necesidades de formación de las maestras del nivel inicial con el fin de promover cambios positivos en la metodología que utilizan.

La propuesta fue validada teóricamente a través del método Delphi (consulta a expertos). Según el resultado del método Delphi, el criterio y valoración de los expertos consultados la propuesta es MUY ADECUADO por tanto es confiable y puede ser aplicado.

CONCLUSIONES

Los aportes más importantes del diagnóstico se presentan a continuación, estos hallazgos son la guía para diseñar el programa de capacitación de acuerdo a las necesidades detectadas en el personal docente y en los niños del Jardín de Infantil de Acción Social "A" con respecto al diagnóstico y atención de la dislalia.

Casi la totalidad de los niños de la muestra comete algún tipo de error en la pronunciación y solo existe una cantidad 
mínima de niños que ya ha superado con éxito los problemas de pronunciación que son propias de la dislalia evolutiva. Sin embargo, es importante resaltar que la mayoría de estos niños comete errores que están dentro del parámetro normal para la edad en la que se encuentran.

Aunque la mayoría de los niños que comete errores están dentro del parámetro normal para la edad en la que se encuentran es importante intervenir para prevenir dislalias funcionales.

También hay niños con retraso en su desarrollo fonológico que deben ser atendidos lo antes posible para evitar que estos niños afiancen los errores de pronunciación que cometen y un porcentaje mínimo presenta deficiencia que es evidente en comparación al desarrollo fonológico de los otros niños de su misma edad. Estos niños que presentan deficiencia evidente son los que más requieren de atención y ayuda de la familia y de la escuela ya que por sí solos no lograran superar los problemas de pronunciación que presentan.

Esta situación problemática genera necesidades de formación en las maestras que trabajan con el nivel inicial puesto que el $100 \%$ de las maestras no conoce y no aplica ningún test para diagnosticar los problemas de pronunciación, así mismo es importante resalta que existen maestras que desconocen completamente actividades que buscan conseguir una base de maduración previa en discriminación auditiva, motricidad bucofacial, respiración y relajación y a pesar de que también hay maestras que conocen al respecto por la complejidad del tratamiento de los problemas de pronunciación ese conocimiento es insuficiente y solo un porcentaje mínimo tiene amplio conocimiento al respecto, algo similar ocurre en relación al conocimiento que poseen en cuanto actividades que ayudan a corregir y afianzar la correcta pronunciación de los fonemas.
Puede concluirse que el conocimiento que las maestras poseen sobre el diagnóstico, prevención e intervención de las dificultades de pronunciación es insuficiente.

Fundamentado en estos resultados se plantea un programa de capacitación para las maestras del nivel de educación inicial para desarrollar competencias en el diagnóstico e intervención de la dislalia para desarrollar procesos de estimulación del lenguaje en su componente fonológico en niños del JIASA.

\section{REFERENCIAS}

Aguilera A. (2004) introducción a las dificultades del aprendizaje. España: Mc Graw-Hill

Almenara, J. C., y Moro, A. I. (2014). Empleo del método Delphi y su empleo en la investigación en comunicación y educación. Edutec. Revista Electrónica de Tecnología Educativa, (48)

Alonzo P. (2010) La dislalia. Rev. Artista digital [Revista en línea], (2) [6 paginas] disponible en: URL:http//www.afapna.es/web/aristadi gital/archivos.../2010_noviembre_21.pdf [Consulta: 2015, Julio 2]

Ausubel D., Novak J. y Hanesian H. (1997).

"Psicología educativa. Un punto de

Castejón J. L. y Navas L. (2011). Dificultades y trastornos del aprendizaje y del desarrollo en infantil y primaria. Alicante, España: Club universitario

Estado Plurinacional de Bolivia. (2009). Constitución Política de Bolivia

Hernández, R, Fernández, C. y Baptista, P. (2010). Metodología de investigación. México: McGraw Hill Interamericana

Hurtado de Barrera, J. (2012). Metodología de la investigación, guía para una comprensión holística de la ciencia. Bogotá: Ediciones Quirón - Sypal.

Jiménez J. y Obispo, J. (2006). Superar las dificultades del lenguaje. Madrid, España: La Tierra Hoy

Lima H. (2004). Cómo ayudar a niños con problemas de aprendizaje. México DF, México: Selector 
Moreno R. y Ramírez Á. Las habitaciones de la dislalia. Rev ReiDoCrea [Revista en línea] 2012; 1 (5): [8 paginas]. Disponible en: URL:http//.www.ugr.es/ miguelgr/ReiD oCrea-Vol.1-Art.5-Moreno-Ramirez.pdf [Consulta: 2015, Julio 2]

Pascual P. (1988) La dislalia. Naturaleza, diagnóstico y rehabilitación. Madrid, España
Pavez, M.; Maggiolo, M y Coloma, C. (2009).Test para evaluar procesos de simplificación fonológica. (3a. ed.) Santiago de Chile: Universidad Católica de Chile

Peña Casanova, J (2005). Manual de logopedia. Barcelona: Masson vista cognitivo. México: Trillas 